\title{
Supporting Grid-based Clinical Trials in Scotland
}

\author{
Prof. R.O. Sinnott, A. J. Stell, O. Ajayi \\ National e-Science Centre, \\ University of Glasgow, \\ United Kingdom \\ r.sinnott@nesc.gla.ac.uk \\ a.stell@nesc.gla.ac.uk \\ o.ajayi@nesc.gla.ac.uk
}

\begin{abstract}
As clinical trials and medical population studies grow in size and complexity, a computational infrastructure that can underpin such studies is highly desirable. This should address several key requirements: it should allow access to and usage of a range of distributed clinical data sets; it should support the efficient processing and analysis of the data obtained; it should have security at its heart and ensure that authorised individuals are able to see data that they are privileged to see and no more. Each clinical trial will have their own requirements on data sets and how they are used, hence a reusable framework that can support a variety of clinical trials and epidemiological studies offers many advantages. The MRC funded Virtual Organisations for Trials and Epidemiological Studies (VOTES) project is a collaborative project involving several UK universities specifically exploring this space. This paper presents the experiences and challenges in developing the Scottish component of this nationwide infrastructure, by the National e-Science Centre (NeSC) based at the University of Glasgow, and the inherent issues brought about by accessing and using the clinical data sets in a flexible, dynamic and secure manner.
\end{abstract}

Keywords

Clinical Trials, Data Federation, Grid

\section{Introduction}

Medical treatments, technologies and other interventions, designed to help improve quality of life, must undergo rigorous assessment before they can be distributed to the population at large. As such, clinical trials are a mandatory requirement to carry out such assessments. To ensure the effectiveness of these trials and studies, the numbers of participants must be high enough to guarantee the strict control of bias (which, in general, requires proper randomization and appropriate analysis) and strict control of random error (which, in general, requires large numbers of relevant outcomes). Generating this evidence typically requires the collection of data on many thousands of people often over periods of several years. Similarly, observational studies assessing the impact of particular exposures (e.g. cigarette smoking) to important clinical outcomes can require large numbers of such events during prolonged follow-up, in order to avoid misleading results caused by statistical anomalies.

The conduct of large-scale randomized trials or observational studies usually requires a collaborative effort, in which data are collected from individuals and from existing health records at multiple investigative sites, and study progress, data quality and analysis of results are managed by one or more coordinating centres.

Grid technology provides one way in which remote, heterogeneous clinical data sets that are often managed by numerous independent bodies, can be seamlessly brought together. A Grid can be defined as a software infrastructure (including computer systems and data storage resources) that enables flexible, secure, co-ordinated resource sharing among dynamic collections of individuals, institutions and resources [1]. 


\section{Grids and Clinical Trials}

For clinical trials and observational studies, the particular attractions of a Grid-based approach are the ability to create, and subsequently manage, virtual organisations (VOs). VOs provide frameworks through which the rules associated with the participants and resources are agreed and enforced. This may well involve agreements upon the remote resources themselves (databases, repositories and the data sets contained therein) and the services that they agree to make available to one another.

The domain of large-scale clinical studies provides special challenges in the level of granularity, flexibility of access and data sensitivity, which lead to the specific labelling of virtual organisations in this context as clinical virtual organisations (CVOs). In particular, CVO policies must strongly adhere to the local policy constraints, for example, on data sharing or confidentiality.

Subject to strict ethical, data protection and security constraints, information can be shared between databases established for routine clinical use (such as general practice and hospital records, disease-specific registries, and central registries) and databases established especially for a particular trial or observational study. Appropriate access to such information from routine clinical systems should enable more efficient recruitment and more complete follow-up of participants in large-scale clinical studies to be achieved more economically.

Furthermore, combining up-to-date data on study participants from both routine and research systems, will allow organisers of clinical trials and observational studies to conduct effective and efficient monitoring of these studies, so that potential problems are identified early. This is all in addition to the advantages of high-availability, reliability, scalability and efficiency that Grid infrastructures provide.

\section{VOTES Infrastructure}

The Virtual Organisations for Trials and Epidemiological Studies (VOTES) project [2] began in October 2005 and has been funded by the MRC for three years to establish such a reusable Grid framework which will support three key stages of any clinical trial or observational study:
- Patient recruitment - attempting to identify eligible candidates, who are willing to participate in a particular trial;

- Data collection - analysing the follow-up data to ensure the proper conduct of a trial, or to identify incidents relating to the treatment itself;

- Study management - identifying the ethical basis of the trial, ensuring that individual policies are being adhered to, and attempting to improve the efficacy of the trial, were it to be repeated.

To meet these demands, it has been recognised that the VOTES framework itself needs to be comprised of specifically engineered collections of adaptable services whose usage and combinations will depend upon the needs of the particular clinical study to support different flavours of CVO.

This infrastructure is envisioned to be distributed throughout the collaborative partners across the VOTES sites and their affiliate resources. This core infrastructure should offer generic Grid services for security, data access and management, and data movement between repositories (peers) hosted at the partner and collaborating institutions.

It is planned that this core infrastructure be expanded and refined to support CVOs that include external peers of two general classes:

- Routine repositories - such as those held by general practices, hospitals, disease-specific registries, device registries, or the Office for National Statistics.

- $\quad$ Study Repositories - developed for a particular trial or observational study.

External peers will apply their own security policy, and may be intermittently connected to the core framework. Though the final result of this dynamic configuration will be a flexible and on-demand system, interfacing with the routine repositories will be a highly involved and potentially politically sensitive process. One of the key areas of VOTES is in the nonIT related issues of ethics in this domain. These will (must) guide the framework, the components it consists of and how they may be applied to support different trials. 
Usage of the infrastructure developed through a collection of integrated local policies requires that all policies are strictly adhered to. An example policy that a CVO framework would be expected to support would be:

"For a specific trial, a study clinician working at the national coordinating centre is allowed to see all trial data (except treatment allocation) for all participants in that country, but only summary data (e.g. a recruitment graph) for participants in other countries."

This policy can be expressed in programmatic terms using a variety of methods - such as XML or through a relational database implementation.

A query not fulfilling all the terms or agreements defined by the CVO policy will be rejected at a given resource and the information associated with the query (the sender, nature of the request, etc) will be logged to enable this to be followed up, including a review of the implementation of the policy itself.

Each CVO resource will have its own local policies, capturing information about who may run queries against them, and what type of queries or data sets can be extracted from them. For some external resources, such information might be implicit (e.g. only a GP is allowed to submit a query against their database), and local policy-based access control software might not exist.

The local policies can be aggregated by subscribing to an over-arching policy for the entire CVO, agreeing to what resources can be accessed by remote users and what are restricted to local users, or other customized fine-grained restrictions as defined by the local resource manager.

Usage of Grid services often requires appropriate adaptors to facilitate access and usage of specific data resources. Such Grid middleware should provide facilities to access and use a variety of database technologies, matching the heterogeneity of resources in clinical trials. The OGSA-DAI [3] project provides mechanisms for managing, accessing and integrating XML relational and file data held in a variety of different databases (DB2, Oracle, MySQL, Xindice, etc) via the Grid.

The OGSA-DAI technology is currently being used to support access to, and storage of, the clinical data, as well as the extensive structured metadata needed to organise data associated with clinical repositories. These metadata will typically describe how, when and by whom the data were produced, and will also describe the data structure and other salient features. The precise description of meta-data will facilitate the ability to conveniently find, create, store, access, integrate and subsequently analyse data from heterogeneous sources.

\section{Scottish Clinical Data Sets, Services and Infrastructure}

One of the immediate key challenges that must be addressed in developing this framework is gaining access to appropriate data sets. Key sources of data in Scotland include national census data sets such as the General Register Office for Scotland [4] which includes information such as the registration of births, marriages, deaths as well as being the main sources of family history records.

The access to such information whilst useful does not include direct health related information which will likely impact upon the suitability of patients to a trial. Primary care and secondary health care data sets are other immediate choices, though access to and usage of these data sets will almost always require ethical approval.

Patients should have the opportunity to consent that their data can be accessed and used. However in running a clinical trial, it is often the case that statistical information is enough. Thus rather than disclosing information on specific patients, statistical information is sufficient. Even here however, questions on ethics are raised. At the very least, doctors and their patients need to be included in any data access decisions.

The focal point of the primary and secondary care data sets being considered thus far within VOTES are based upon discussions with the NHS Information Services [5] and their associated technology providers. These include:

- The General Practice Administration System for Scotland (GPASS) [6] which is the core IT application used by over $85 \%$ of clinicians and general practitioners involved in primary care across Scotland.

- Scottish Morbidity Records (SMR) [7] - which includes good quality (linked) records relating to a variety of patient information records, 
compiled primarily from hospital visit records across Scotland.

- $\quad$ Scottish Care Information (SCI) Store [8] - a batch storage system which allows hospitals to add a variety of information, e.g. lab results, to be shared across the community. Currently there are 15 different SCI Stores across Scotland. Each of these SCI Store versions has their own data models, and schemas, based upon the regional hospital systems they are supporting.

It is the case that these solutions are currently undergoing a process of evolution across the NHS in Scotland. Commercial suppliers are producing software under contract by the NHS, yet it is currently the case that a fabric or overall systems architecture which will be used to integrate all of these software families and their associated data sets has not been fully defined. Thus for example, different sources of data and services use different classification schemes such as International Classification of Disease version 10 (ICD-10) [21], as well as ICD version 9 and other older Read coding schemes.

Similarly, systems such as the SCI Store laboratory systems often use different patient identifiers. For example SCI Store supports locally controlled cross-matching to ensure an incoming piece of information is attached to the correct patient's record. This crossmatching is in turn based on a link to one or more local patient administration systems and the national Community Health Index (CHI) number patient identifier, equivalent to England's National Health Service (NHS) number.

With regard to VOTES, there might be numerous ways that the remote repository data might be integrated with the GPs data however what is clear is that this data should be ethical and electronically verifiable, so that the data has the appropriate type and is within given predictable constraints, e.g. upper and lower limits for a cholesterol level, or for a blood pressure measurement, or for a patients body mass index level etc. The term ethical is italicised here since it is essential to ensure that software solutions are developed for the right reasons and that ethical control of software IT push is ensured.

Thus for example, software solutions which allow laboratory test results to be automatically incorporated into the primary care record without the GP's knowledge could be envisaged. Such an approach could be considered to be of use however this would be potentially very dangerous and clinically unacceptable since the potential possibility for errors always exists. Hence, interfaces and associated causalities are needed which can facilitate user/clinician driven control which is in the best interest of the patient at all times.

\section{Infrastructure Challenges}

The main challenges in building a framework that will dynamically and securely realise the benefits of Grid technology can be broadly broken down into three categories: (1) data and object classification, (2) security and (3) ethics.

\subsection{Classification}

As CVOs necessarily span heterogeneous domains, a pre-requisite to the construction of distributed queries and aggregation or joining of data returned is the development and use of a standard method of classification or common vocabulary more generally.

There are two widespread solutions to this problem, which both have advantages and disadvantages:

- Global schema - this would act as an over-arching descriptive structure that all parties would subscribe to. Whilst providing a readily available standard, the solution is static and would encounter problems when new clinical resources with new and different schemas were to be added. The schema would run the risk of becoming large and unwieldy.

- Ontology - a common vocabulary and agreement on concepts and their relationships that allows differing schemas to understand the nomenclature for similar objects. This would be more flexible than a single schema. However it still requires a large network of relationships to be defined between each of the schemas, giving rise to a large and un-maintainable system.

One key challenge in this is in understanding the currently used classifications and schemes and models that will have some future longevity. Thus there exist a broad range of different standards and data classifications that exist today across the e-Health domain including efforts such as Health-Level 7 (HL7) 
[9], SNOMED-CT [10] and OpenEHR [11]. All of these and the ICD-10, ICD-9 and ReadCoding classifications are used across the NHS along with many other solutions such as DICOM [12] also in existence.

It is possible to develop Grid based solutions which will allow access to and usage of data sets existing in or supporting all of these classifications, however the effort required to do so is considerable. The HL7 data model and information schema for example, appears to offer much functionality, but at the cost of complexity. It is also not clear precisely how widely supported the HL7 standards are in the NHS across Scotland. Instead the focus of VOTES currently is looking at those more empirical data sets associated with SMR, GPASS and SCI Store.

However the eventual solution manifests itself, the need for a common understanding of data classification is paramount, as it is a necessary pre-requisite to implementing distributed security models. Only once the nature of the objects within a structure is properly understood, can that structure be properly secured.

\subsection{Security}

Information stored in clinical trials is by its nature, highly sensitive - drug treatments, conditions and diseases that patients have must be kept in the strictest confidence and the exact details should only be known about by a few privileged roles in the trial. This is one of the most fundamental challenges in this work - to realise the opportunities and benefits that can be brought to this field by Grid technology but to also maintain the high security standards that must be strictly adhered to.

Within the Grid community security issues are generally grouped into the categories of:

- authentication - the discovery and checking of a user's identity ("Who are you?")

- authorization - the discovery and enforcement of that user's privileges based on their identity ("What are you allowed to do?")

- accounting - logging the activity of users so that they can be held accountable for their actions within a system. ("Where were you on the night of...?”)
In addition to the "AAA" security aspects defined above, another artefact of security that is essential in the clinical domain is that of "anonymisation". This process involves allowing less-privileged users to gather statistical data for the purposes of studies or trials, but without revealing the associated identifying data - this only being available to users with greater privileges.

The NHS in Scotland currently achieves this by encrypting a unique number associated with all patients across Scotland: the CHI number. Once an anonymised patient has been matched for a clinical trial, this encrypted value can in principle be sent to the Practitioners Service group [13] of the NHS who will, as one of the many services that they provide, decrypt it and contact the patients directly (assuming ethical permission has been granted for so doing) to ask if they wish to join the clinical trial.

A further challenge is that there are currently many independent solutions across the NHS for how they manage their infrastructures. The result is that many idiosyncratic encryption solutions exist across the different health boards, to achieve this anonymisation.

A proposed solution to this is to develop a semi-centralised Anonymisation Service, analogous to a Certification Authority or any other implementation of third-party trust model, which would gather the results of the distributed query and anonymise the identifying data before sending it back to the user (dependent on their privileges). This would assume a super-user type of privilege for the Anonymisation Service [22].

However, the corollary notion of statistical inference - where the identity of an individual could be deduced by comparing and combining separate items of non-identifying data - must also be addressed. The solution most likely to protect against this would be to set a minimum threshold of record numbers, which a query must return more than, else no records are returned at all.

A security technology that is rapidly gaining ground in the academic and Grid communities is that of Shibboleth [14], which supports the process of enforcement of authentication and authorization between institutions participating within a federation. Shibboleth relies on local authentication and release of security attributes associated with that individual when attempts to access inter-institutional resources are made. Remote resource providers are fully 
autonomous and can define whether this information is sufficient to allow access to their own local resources for example. This model improves existing Grid based authentication schemes since it builds upon local interactions and knowledge of users.

This ability to authenticate and authorize users within a federation of partners is well-suited to the description of the issues of providing security within a distributed CVO.

\subsection{Ethics}

Throughout the VOTES project, continuous ethical and legal overview of the solutions being put forward and the data sets accessed are being made. This includes the perceived benefits of the research for the public, and is undertaken by independent ethical oversight committees. To support this, superior security roles for oversight committee members which allow access to all data sets and reports for given clinical trials will be made available.

Many of the data access and security challenges depend upon the notion of consent. If a patient is willing that their data sets can be made available for research purposes then this greatly simplifies the ethical considerations which constrain the design space. Within initial implementations in VOTES, various models of obtaining patient consent have been investigated.

A key element in the realisation of the live system, with ethical viability, is trust: trust of the technology and trust of the people and processes. To support this it is planned that the NeSC Glasgow researchers will be given honorary contracts to work at the NHS in Glasgow.

Through immersing themselves in the NHS environment, a better understanding of the daily processes for data production, data linkage and management and general software IT support will be garnered. It is clear that to have any chance of wide scale uptake, the Grid cannot subsume existing infrastructures and practices. Instead, the Grid framework and associated solutions must be constrained to function within the context of existing solutions and practices.

\section{VOTES Implementation}

Due to the sensitive nature of the data involved, and the resultant security and ethical concerns outlined above, the development of any infrastructure that queries such data, must itself be rigorously tested and evaluated within a contained environment.

To this end, initial solutions have been developed on a test-bed of local databases representative of the types and structures of data repositories currently used by the NHS in Scotland already. The data itself is constituted of dummy (but representative) data sets that represent typical clinical data whilst not actually being "live". In this way, applications can be developed that can test different models of data access and retrieval whilst being aligned with ethical protocols.

Several models are currently being considered, in anticipation of the system being adopted for use with such "live" data:

- A "push" model, where vetted (anonymised) data is exported to the Grid community for use within the trial recruitment querying phase.

- A controlled push model where data is made available on a resource within a demilitarised zone in the NHS for secure access/retrieval by Grid services.

- Letting GPs and clinicians drive the recruitment process by installing the Grid software within the NHS domain.

All of these models depend on a level of trust existing between the parties involved.

\subsection{Architecture}

The basic architecture which supports federated queries in a user oriented but secure manner is depicted in Figure 1. This Grid infrastructure is hosted at the Glasgow National e-Science Centre.

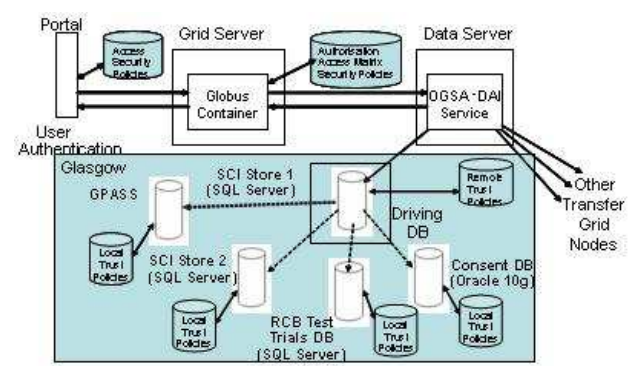

Figure 1: Architecture of core Grid infrastructure as detailed for the Glasgow node of the CVO. The branches pointing out of the data server box indicate where the connection to other CVO nodes would occur. 
The modus operandi of the system is as follows:

- The user logs in to a GridSphere [15] portal either directly or via Shibboleth (at their home institution Identity Provider). The roles and privileges associated with that user are used to configure the portal and services, and subsequently data that can be accessed.

- They then select a particular clinical trial. This communication is passed between the portal and a grid service coded using the Globus Toolkit v4.0 [16].

- The parameters valid for this role and trial are returned to the user, where they are allowed to make a selection and apply a relevant condition.

- A distributed SQL query is put together based on this selection, and the databases relevant to that particular trial are joined using a common index (in this case, the $\mathrm{CHI}$ number).

- The results are passed through an OGSA-DAI service to allow database querying using a SOAP interface, with the results presented as standard XML.

- $\quad$ The XML is tidied up and presented to the user as one single resource.

The query run from the "driving database" is distributed over the pool of databases for which it is responsible, the results from which are wrapped in a standard SOAP message, passed back through the data and grid servers to the portal. In this scenario, the driving database effectively acts as a custodian of the local database resources, which ultimately provide the pertinent clinical data.

By use of the Shibboleth technology, attributes providing the relevant privileges within the context of specific trials are transparently (from the user perspective) returned from the Identity Provider. At Glasgow the Identity Provider is an LDAP directory holding the attributes for each user local to that institution - and these are used to bring back the appropriate privileges for a specific trial. The university is also moving towards a unified account management system which will have a one-one relationship between staff, students and their associated privileges. This is being explored within the GLASS project [20].

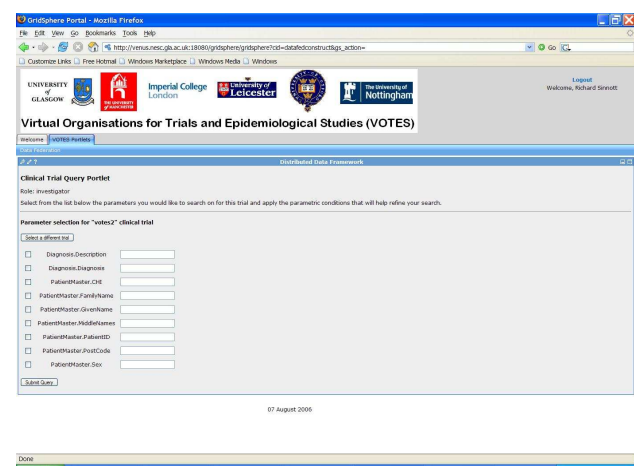

Figure 2: the parameters available for query when the "investigator" role attempts to find information about the "votes2" trial.

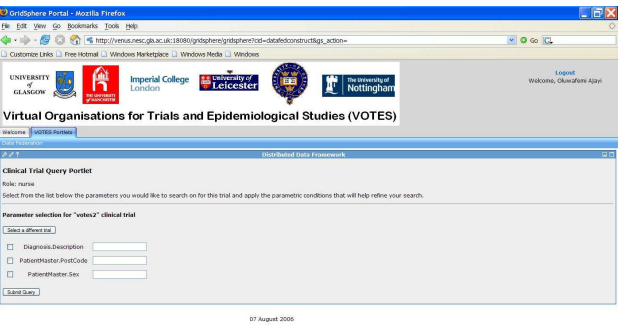

Figure 3: the parameters available for the same trial, but this time requested by the nurse. Note that the parameters only refer to non-identifying data such as postcode, sex, etc.

Figures 2 and 3 show two screen-shots that highlight the difference between the parameters returned for the same trial, when requested by roles of differing privileges.

Through the use of this application, the end user is able to seamlessly access a set of resources, pertinent to clinical trials, in a dynamic, secure and pervasive fashion. They are simply required to sign-in (authenticate) once at their home institution. Depending on the user's privileges, the results returned have varying degrees of verbosity thereby allowing limited statistical analysis without compromising the privacy restrictions necessarily applied in such sensitive data.

Figure 4 shows the results returned from a distributed query, presented as one resource to the end-user. Note the images in that figure are of brain MRI scans, part of the BrainIT project [19], just one project that would benefit significantly from the VOTES infrastructure. In addition a rich variety of physiological data sets on the patients is also returned such as the nature of their Brain trauma; treatments they 
are receiving; real time monitoring information and a variety of other clinical information.

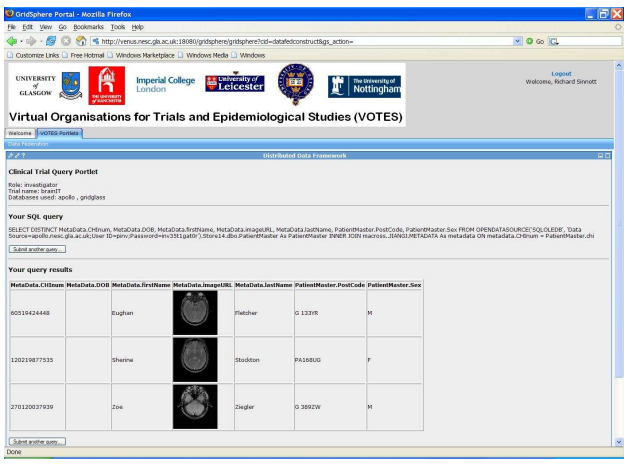

Figure 4: Results returned from distributed query.

\subsection{Security Framework}

In the current implementation, data federation security is achieved at both local and remote level. The local level security, managed by each test site, filters and validates requests based on local policies at DBMS levels. The remote level security is achieved by the exchange of access tokens between the designated Source of Authority (SoA) at each site. These access tokens are used to establish remote database connections between the sites in the federation. In principle local sites authorise their users based on delegated remote policies.

As the aggregation of authorization privileges is compiled before run-time, the interface presented to the end-user allows them to enter queries that will not fail (i.e. the parameters definitely exist throughout the distributed resources and the correct syntax will be submitted). An alternative explored was to allow users to input customised SQL queries. However this assumed a knowledge of the schema that clinicians may not have, and in fact, will definitely not have for arbitrary remote resources. A key issue to be considered is what information should be presented to clinical trial investigators since the schemas for the federated resources are complex typically over several hundred tables.

The infrastructure presented depends on a secondary framework existing that allows to dynamically create access policies for different trials and resources. The notion of having administrative super-roles that can set these policies has been investigated and it is believed that two distinct roles are needed in this space:
- A trial administrator - learned in clinical practices and responsible for creating and setting up policies for specific trials.

- A node administrator - responsible for the underlying Grid infrastructure resources and their interface communicating with the other nodes within the CVO.

With these two roles in place it will be possible to realise a truly dynamic and secure system.

\section{Conclusions and Future work}

The VOTES prototype software is very much a work in progress. Yet the experiences in developing this prototype are helping to gain a better understanding of the clinical domain problem space and shaping the planned Grid framework.

The vision of a Grid framework eventually supporting myriad clinical trials and epidemiological studies is a compelling one, but can only be achieved once experiences have been gained in accessing and using a wide variety of clinical data sets. In achieving this, it is immediately apparent that there are a number of political and ethical issues that must be addressed when dealing with data-sharing between domains and these are inherently more difficult to deal with than the technological challenges.

Currently the prototype system supports a variety of models which are allowing exploration of the potential solution space for patient consent across Scotland. For example solutions have been prototyped which allow patients to consent to their data being used: for a specific clinical trial, for a particular disease area, consent for their data being used generally.

In addition, it will also be necessary for the system to allow for patients to opt out, i.e. their data sets may not be used for any purposes. Numerous variations on this are also being explored, e.g. the patients' data may only be used provided they are contacted in advance.

In terms of the back-end repositories, whilst the NHS in Scotland and the UK more widely are taking steps to standardise the data-sets that they have, these are still far from being fully implemented (and accepted) by clinical practitioners. For instance, the unique index reference number the Community Health Index 
has only been implemented across some regions of Scotland and therefore leaves certain areas with incomplete references.

Those records that do not have the CHI number are referenced using a different Patient Identification (PID) number that will be idiosyncratic to the region in question. There is also a need to build up trust relationships with the end-user institutions that we are working with to support this clinical infrastructure. This necessarily takes time and will be furthered by engaging in an exchange program where employees from NeSC work with and understand the processes in the NHS IT departments and vice-versa.

A second project involving computerised access to MRI brain scan images and data is currently being undertaken at the National eScience Centre, in collaboration with the Glasgow Southern General Hospital [20]. The potential for alignment with the prototype system being developed in VOTES was quickly recognised and the framework developed was easily modified to query and return this data (also illustrating the flexible nature of the framework).

This overlap is indicative of the widespread relevance of this kind of infrastructure. With this in mind, the applications described here are being developed with a view to being rolled out to the NHS Scotland in the first instance, moving from test data to "live" data with fully audited and standards-compliant security, upon establishment of reliability and production value. The eventual vision is that this infrastructure will one day be available on a global scale allowing health information to be exchanged across heterogeneous domains in a seamless, robust and secure manner. In this regard, we are currently exploring international collaborative possibilities with the caBIG project in the US [17] and closer to home in genetics and healthcare projects across Scotland [18].

\section{References}

[1] The Anatomy of the Grid: Enabling Scaleable Virtual Organisations - Foster, Kesselman, Tuecke

[2] VOTES -

http://www.nesc.ac.uk/hub/projects/votes

[3] OGSA-DAI - http://www.ogsadai.org.uk

[4] GRO for Scotland - http://www.gro-

scotland.gov.uk

[5] NHS Information Services Division http://www.isdscotland.org
[6] GPASS - http://www.gpass.co.uk

[7] Scottish Morbidity Records -

http://www.show.scot.nhs.uk/indicators/SMR/

Main.htm

[8] SCI Store -

http://www.show.scot.nhs.uk/sci/products/store

[9] HL7 - http://www.hl7.org/

[10] SNOMED-CT -

http://www.snomed.org/snomedct/

[11] OpenEHR - http://www.openehr.org/

[12] DICOM - http://medical.nema.org/

[13] Practitioners Services Group http://www.psd.scot.nhs.uk/

[14] GridSphere - http://www.gridsphere.org

[15] Globus - http://www.globus.org

[16] Shibboleth -

http://shibboleth.internet2.edu

[17] caBIG - https://cabig.nci.nih.gov/

[18] Scottish Family Health Study -

http://www.innogen.ac.uk/Research/The-

Scottish-Family-Health-Study

[19] Personal Communication - Ian Piper,

Glasgow Southern General Hospital

[20] Glasgow early adoption of Shibboleth

(GLASS) project,

www.nesc.ac.uk/hub/projects/glass

[21] International Statistical Classification of

Disease and Related Health Problems (ICD) -

http://www.connectingforhealth.nhs.uk/clinical coding/classifications/icd_10

[22] Stell, Sinnott, Ajayi - Secure Federated

Data Retrieval in Clinical Trials, TeleHealth

Conference 2006, Banff, Alberta 


\section{University Library}

\section{- M M I E E R VA A gateway to Melbourne's research publications}

Minerva Access is the Institutional Repository of The University of Melbourne

Author/s:

Sinnott, R. O.;Stell, A. J.;Ajayi, O.

Title:

Supporting grid-based clinical trials in Scotland

Date:

2008

Citation:

Sinnott, R. O., Stell, A. J., \& Ajayi, O. (2008). Supporting grid-based clinical trials in Scotland. Health Informatics Journal, 14(2), 79-93.

Publication Status:

Published

Persistent Link:

http://hdl.handle.net/11343/28770 